\title{
$\mathcal{P}$ T-symmetric Quantum Chain Models
}

M. Znojil

A review is given of certain tridiagonal $N$-dimensional non-Hermitian J-parametric real-matrix quantum Hamiltonians $H^{(N)}$. The domains $\mathcal{D}^{(N)}$ of reality of their spectra of energies are studied, with particular attention paid to their exceptional-point boundaries $\partial \mathcal{D}^{(N)}$. The strongest admissible couplings are specified in closed form for all $N$.

Keywords: quantum mechanics, pseudohermitian Hamiltonians, $\mathcal{P}$ T-symmetric nearest-neighbor interactions, exactly solvable finite-dimensional models, domains of quasihermiticity, exceptional points.

\section{Introduction and summary}

A more or less satisfactory explanation of the numerous experimental observations that the states of molecules, atoms and atomic nuclei are quantized belongs to the most important achievements of physics made, predominantly, during the first few decades of the twentieth century [1]. It is, therefore, slightly surprising that the situation is still not yet entirely satisfactory at present. In particular, one often has to rely upon phenomenological models in nuclear physics where our uncertainties concerning the "correct form" of the interactions between individual nucleons are combined with the enormous mathematical difficulties arising in connection with a sufficiently reliable numerical solution of the underlying quantum-mechanical many-nucleon problem.

One of the ways out of the latter theoretical as well as practical trap has been found in the elimination of as many irrelevant degrees of freedom as possible. Perceivable success has been encountered in the so called interacting boson models, where practical solvability of the complicated (i.e., partial differential or integro-differential) linear Schrödinger equations for bound states,

$H\left|\psi_{n}\right\rangle=E_{n}\left|\psi_{n}\right\rangle, \quad n=0,1, \ldots$

has been achieved via their reduction to the "effective", simplified form

$H^{(e f f)}\left|\psi_{n}^{(e f f)}\right\rangle=E_{n}^{(e f f)}\left|\psi_{n}^{(e f f)}\right\rangle, \quad n=0,1, \ldots, n_{\max }$,

where $H^{(e f f)}$ is a finite-dimensional and, often, real and symmetric matrix.

In the latter context as briefly reviewed, e.g., in [2], a conflict survives between the numerical reliability and practical tractability of the effective models. In the context of the so called Dyson-mapping approximation technique, for example, it was originally felt as an unpleasant surprise that the requirement of the smallness of the dimension of the matrix $H^{(e f f)}$ emerged in an apparently inseparable combination with the necessity of moving to a less standard Hilbert space $H^{\text {(physical) }}$ of states where the definition of the inner ("scalar") product between elements $|\chi\rangle \in H^{\text {(physical) }}$ and $|\xi\rangle \in H^{\text {(physical) }}$ had to be modified,

$$
\langle\chi \mid \xi\rangle \rightarrow\langle\chi|\Theta| \xi\rangle, \quad \Theta=\Theta^{\dagger}>0
$$

This trick very easily extends the applicability of the formalism of standard textbook Quantum Mechanics by the transition to the various nontrivial, "non-Dirac" metric operators. Of course, all the observable quantities must be then represented by the operators $\mathcal{O}^{\text {(physical) }}$ which are self-adjoint in $\mathcal{H}^{\text {(physical) }}$

Whenever one chooses a nontrivial metric $\Theta^{\text {(physical) }} \neq I$ in $\mathcal{H}^{\text {(physical) }}$, all the operators $\Theta^{\text {(physical) }}$ which are self-adjoint in $\mathcal{H}^{\text {(physical) }}$ must obey the consistency condition

$$
\left[\mathcal{O}^{(\text {physical })}\right]^{\dagger}=\Theta \mathcal{O}^{(\text {physical })} \Theta^{-1} \text {. }
$$

Unfortunately, confusion may (as it often does [3]) immediately arise in all the models where one employs, in parallel, another, auxiliary but much more easily tractable Hilbert space $\mathcal{H}^{\text {(unphysical) }}$, the scalar product in which is specified by the unit metric $\Theta^{\text {(unphysical) }}=I$. For this reason, it has been recommended [2] to call the operators $\mathcal{O}^{\text {(physical) }}$ [of eq. (2), with $\Theta \neq I$ ] "quasi-Hermitian", while reserving the name "Hermitian" solely for the subset of operators $\mathcal{O}$ for which eq. (2) holds at the traditional "Dirac" special metric $\Theta=I$.

The latter convention is believed to minimize the possible misunderstandings, in spite of the apparently counterintuitive fact that all the operators of observables $\mathcal{O}^{\text {(physical) }}$ may be called, strictly speaking, "non-Hermitian", at least from the point of view of the more conventional, albeit auxiliary, Hilbert space $\mathcal{H}^{\text {(unphysical) }}$. The latter, apparently innocent-looking paradox demonstrated its full strength and impact when Bender and Boettcher published their apparently surprising observation [4] that certain remarkably elementary (viz. ordinary differential) Hamiltonians $H^{(B B)}$ possess, in spite of their manifest non-Hermiticity (with respect to the most common Hilbert space $L_{2}(-\infty, \infty)$ ), real and discrete (i.e., bound-state-like) spectra.

It took a few years before the Bender's and Boettcher's apparent puzzle was resolved. Independently, several groups of people realized that in our present language and for all the models in question we have $L_{2}(-\infty, \infty) \equiv \mathcal{H}^{\text {(unphysical) }}[5$, $6,7,8,9,10,11,12,13,14]$. This means that inside a certain quasi-Hermiticity domain $\mathcal{D}$ of parameters where the energies remain real $[4,7]$, all the models $H^{(B B)} \neq\left[H^{(B B)}\right]^{\dagger}$ do satisfy the necessary quasi-Hermiticity condition (2). One even 
realizes [2], [10] that there exist quite a few different metrics $\Theta=\Theta^{\text {(physical) }} \neq I$ which can all be assigned to the "candidate-for-the-Hamiltonian" operator $H \neq H^{\dagger}$.

In what follows we intend to return to the problem of the ambiguity of the assignment of an "optimal" $\Theta^{\text {(physical) }}$ to a given $H \neq H^{\dagger}$. For the sake of clarity we shall restrict our attention to the effective-Hamiltonian models described by the following real matrices of the chain-model form,

$H^{(N)}=\left[\begin{array}{cccccc}1-N & g_{1} & 0 & 0 & \cdots & 0 \\ -g_{1} & 3-N & g_{2} & 0 & \cdots & 0 \\ 0 & -g_{2} & 5-N & \ddots & \ddots & \vdots \\ 0 & 0 & -g_{3} & \ddots & g_{2} & 0 \\ \vdots & \vdots & \ddots & \ddots & N-3 & g_{1} \\ 0 & 0 & \cdots & 0 & -g_{1} & N-1\end{array}\right]$.

Complicated as the problem may look for the general $N$, we shall recollect and summarize some results of refs. [15]-[20] and show that, and why, these models remain tractable, up to a large extent, by certain analytic, perturbative or algebraic non-numerical techniques.

After a compact and more or less self-contained review of the underlying physics in Section 2 we shall formulate our project in Section 3. At the first few lowest dimensions $N$, we shall then derive some consequences of the implicit secular-equation definitions of the energy spectra in the respective Sections 4-9, with particular emphasis on non-perturbative, strong-coupling results. Sections 10-12 will finally summarize our observations and, in a climax of our present message, they enable us to conjecture an extrapolation of some of the formulae to all the finite dimensions.

\section{$2 \mathcal{P}$ T-symmetry of the chain model (3)}

Once we assume that all the matrix elements of $\mathrm{H}^{(N)}$ remain real we observe that

$$
\left[H^{(N)}\right]^{\dagger} \mathcal{P}=\mathcal{P} H^{(N)}, \quad \mathcal{P}=\left[\begin{array}{ccccc}
1 & 0 & \ldots & & \\
0 & -1 & 0 & \ldots & \\
\vdots & 0 & 1 & 0 & \ldots \\
& \vdots & 0 & -1 & \ddots \\
& & \vdots & \ddots & \ddots
\end{array}\right]
$$

In the literature the latter formula is usually called $\mathcal{P}$-pseudo-Hermiticity [10]-[13] (or, in the context of physics [4, 21], $\mathcal{P} \mathcal{T}$-symmetry) of $H^{(N)}$. Due to the exceptional simplicity of its "parity-reversal" matrix factor $\mathcal{P}$, the validity of eq. (4) may also significantly simplify an explicit re-construction of $\Theta^{\text {(physical) }}$, proceeding in three steps. In the first step we imagine that $H^{(N)}$ is non-Hermitian so that we may and have to solve not only the standard Schrödinger's linear-algebraic eigenvalue problem

$$
H^{(N)}|n\rangle=q_{n}|n\rangle, \quad n=1,2, \ldots, N
$$

(giving all the right eigenvectors $|n\rangle$ of $H^{(N)}$ as its result) but also the parallel, "left-eigenvector" linear algebraic problem at the same eigenvalue,

$$
\left\langle\langle m| H^{(N)}=\left\langle\langle m| q_{m}, \quad m=1,2, \ldots, N\right.\right.
$$

(note that $\langle n|\neq| n\rangle\rangle$ for the generic $H^{(N)} \neq H^{(N)^{\dagger}}$ ). In the second step we assume that the eigenvalues remain real and non-degenerate (thus, in our notation, all our coupling constants $g_{j}$ stay inside a real quasi-Hermiticity domain $\mathcal{D}$ ) and recollect the following explicit general formula for the metric,

$$
\left.\Theta=\sum_{n=1}^{N}|n\rangle\right\rangle \sigma_{n}\left\langle\langle n|, \quad \sigma_{n}>0\right.
$$

(cf., e.g., [10]-[13]) where any choice of the $N$-plet of the real parameters $\sigma_{n}$ defines an eligible Hilbert space $\mathcal{H}^{\text {(physical) }}$ equipped with the inner product (1).

In the final step we notice that the "additional" problem (5) can be re-written in the equivalent form

$$
\left.\left.H^{(N)^{\dagger}}|n\rangle\right\rangle=q_{n}^{*}|n\rangle\right\rangle, \quad n=1,2, \ldots, N .
$$

Obviously, its solution [i.e., a key assumption of the feasibility of an evaluation of the sum (6)] can be circumvented because in the light of eq. (4) the "unknown" left eigen-ketkets are all proportional to the "known" right eigen-kets multiplied by a diagonal matrix,

$$
|n\rangle\rangle=Q_{n} \mathcal{P}|n\rangle \text {. }
$$

Here the coefficients $Q_{n}$ are arbitrary. Their variability represents in fact the freedom in the normalization of our (as we can prove, biorthogonal [7]) basis (composed of the left and right eigenvectors of $\left.H^{(N)}\right)$. In the case of the real spectrum $q_{n}$ one can easily fix their choice in such a way that the inner product becomes positive definite. Whenever necessary, we may even re-scale their values to $\pm 1,-$ that's why we called these coefficients "quasiparities" in [7].

\section{The extreme exceptional points}

Secular-equation definitions $\operatorname{det}\left[H^{(N)}-E I\right]=0$ of the energies degenerate to the polynomial equations in $E^{2}=s$,

${ }^{J}+P_{J-1}(A, B, \ldots) s^{J-1}+P_{J-2}(A, B, \ldots) s^{J-2}+\ldots=0$.

Here we abbreviated $A=g_{J}^{2}, B=g_{J-1}^{2}, \ldots, Z=g_{1}^{2}$, with $J=$ entier $[N / 2]$. In the $J$-dimensional space of our new, non-negative coupling parameters $A, B, \ldots, Z$, all the spectrum of the energies $E= \pm \sqrt{s}$ remains real only inside a certain compact, hedgehog-shaped domain $\mathcal{D}$.

Our main attention will be paid to the "spikes of the hedgehog", i.e., to the $2^{J}$ points where the boundary $\partial \mathcal{D}^{(N)}$ forms certain protruded spikes. In the context of physics these spikes represent the strong-coupling extremes in the set $\partial \mathcal{D}^{(N)}$ tractable as Kato's exceptional points occurring in the real domain [22].

One can notice that at all these extreme exceptional points (EEPs) [with coordinates $\left.A^{(\mathrm{EEP})}, B^{(\mathrm{EEP})}, \ldots, Z^{(\mathrm{EEP})}\right]$, all the energy levels degenerate to the single value of $E^{(\mathrm{EEP})}=0$. This follows from the up-down symmetry of the unperturbed levels as well as of their perturbations. As a consequence, the EEP secular equation acquires the form $\left(E-E^{(\mathrm{EEP})}\right) N=0$ so that in the light of eq. (8), all of the EEP coupling strengths will have to satisfy the set of the following $J$-plet of polynomial equations, 


$$
\begin{gathered}
P_{J-1}\left(A^{(\mathrm{EEP})}, B^{(\mathrm{EEP})}, \ldots\right)=0, \\
P_{J-2}\left(A^{(\mathrm{EEP})}, B^{(\mathrm{EEP})}, \ldots\right)=0, \\
\cdots \\
P_{0}\left(A^{(\mathrm{EEP})}, B^{(\mathrm{EEP})}, \ldots\right)=0 .
\end{gathered}
$$

Although the simplicity of their solutions is amazing, their derivation is from easy. Let us now study this problem with a step-by-step increase of the dimension.

\section{Two-dimensional model of paper [15]}

In ref. [15] we paid attention to all the $\mathcal{P} \mathcal{T}$-symmetric real matrices

$$
H^{[15]}=\left(\begin{array}{cc}
-1+c & a \\
-a & 1-c
\end{array}\right) .
$$

All of their eigenvalues (i.e., eigenenergies) are known in the closed form,

$$
E= \pm \sqrt{(1+c)^{2}-a^{2}} .
$$

Once we decided to ignore the "pathological" case with $c=c_{\text {path }}=-1$ (giving complex energies), an elementary re-scaling enabled us to put $c=0$ and get the first nontrivial one-parametric version of our present class of models $H^{(N)}$.

Our main result was that one can guarantee the reality of the spectrum in an interval $\mathcal{D}^{(2)}=(-1,1)$ of our couplings $a=\cos \alpha$ with, say, $\alpha \in(0, \pi)$. We also discussed several eligible ways of suppressing the ambiguity of the metric. This task proved easily achieved: We simply extracted the general metric from eq. (2) via a real, Hermitian ansatz

$$
Q=\left(\begin{array}{ll}
t_{1} & t_{2} \\
t_{2} & t_{3}
\end{array}\right) .
$$

This enabled us to reduce all the construction to the single condition

$$
2 t_{3}+\left(t_{1}+t_{2}\right) \cos \alpha=0 .
$$

Two free parameters survived and the latter relation defined the value of $t_{3}$. Once we set $t_{1}=Z(1+\xi)$ and $t_{2}=Z(1-\xi)$ we have $t_{3}=Z \cos \alpha$ as well as the overall-scaling interpretation of $Z[16]$.

Once we had constructed the metric $\Theta$ compatible with eq. (2), it remained for us to guarantee that our $\Theta$ was positive definite. Fortunately, both the eigenvalues of $\Theta$ are available in closed form,

$$
\theta_{ \pm}=Z\left(1 \pm \sqrt{\xi^{2}+\cos ^{2} \alpha}\right.
$$

so that the derivation of the final constraint $\xi^{2} \leq \sin ^{2} \alpha$ is trivial.

\section{Three-dimensional model of paper [17]}

Out of the generic three-by-three model of ref. [17] with two free parameters,

$$
H^{[17]}=\left[\begin{array}{ccc}
2 & a+\xi & 0 \\
-a-\xi & 0 & a-\xi \\
0 & -a+\xi & -2
\end{array}\right]
$$

the present one-parametric chain model $H^{(3)}$ is obtained in the limit $\xi \rightarrow 0$. Thus, the determination of the interval of the quasi-Hermiticity $\mathcal{D}^{(3)}=(-\sqrt{2}, \sqrt{2})$ is trivial since the secular equation $-E^{3}+\left(4-2 a^{2}\right) E=0$ is exactly solvable in closed form.

\section{Four-dimensional model of paper [18]}

The four-dimensional model of paper [18] contains four free real parameters, but it contains our present two-parametric chain model with $N=3$ as a special case. Such a reduction simplifies the $N=4$ secular equation

$$
\operatorname{det}\left[\begin{array}{cccc}
3-E & b & 0 & 0 \\
-b & 1-E & a & 0 \\
0 & -a & -1-E & b \\
0 & 0 & -b & -3-E
\end{array}\right]=0
$$

and makes it equivalent to the quadratic equation for $s=E^{2}$, $s^{2}+\left(-10+2 b^{2}+a^{2}\right) s+9+6 b^{2}-9 a^{2}+b^{4}=0$

solvable in closed form,

$s=s_{ \pm}=5-b^{2}-\frac{1}{2} a^{2} \pm \frac{1}{2} \sqrt{64-64 b^{2}+16 a^{2}+4 b^{2} a^{2}+a^{4}}$.

The discussion of the two-dimensional domain $\mathcal{D}^{(4)}$ of the reality of these energies is entirely analogous to the previous case. Also the set of the polynomial EEP equations remains elementary,

$$
A+2 B=10, \quad(3+B)^{2}=9 A .
$$

The elimination of $A$ leads to a quadratic equation for $B+3$ giving a spurious solution $A=64$ and $B=-27$ (which would imply an imaginary coupling $b$ ) and the unique correct solution $A^{(\mathrm{EEP})}=4$ and $B^{(\mathrm{EEP})}=3$.

\section{Five-dimensional case}

The model

$$
H^{(5)}=\left[\begin{array}{ccccc}
4 & b & 0 & 0 & 0 \\
-b & 2 & a & 0 & 0 \\
0 & -a & 0 & a & 0 \\
0 & 0 & -a & -2 & b \\
0 & 0 & 0 & -b & -4
\end{array}\right]
$$

gives the central constant energy $E_{0}=0$ so that its secular equation

$-s^{2}+\left(20-2 b^{2}-2 a^{2}\right) s-64-16 b^{2}+32 a^{2}-b^{4}-2 a^{2} b^{2}=0$

is solvable in closed and compact form again,

$$
\begin{aligned}
& E_{ \pm 1}= \pm \sqrt{10-a^{2}-b^{2}-\sqrt{36+12 a^{2}+a^{4}-36 b^{2}}} \\
& E_{ \pm 2}= \pm \sqrt{10-a^{2}-b^{2}+\sqrt{36+12 a^{2}+a^{4}-36 b^{2}}}
\end{aligned}
$$


Thus, the triplet of the necessary inequalitites comprises the trivial simplex condition $10 \geq A+B$, condition $36+12 A+A^{2} \geq 36 B$ [showing that $B$ must lie below a parabola $\left.B_{\text {max }}=B_{\max }(A)\right]$ and condition $(8+B)^{2} \geq(32-2 B) A$ [giving the upper bound for $\left.A \leq A_{\max }=A_{\max }(B)\right]$. In the EEP context, two coupled conditions degenerate to the single quadratic equation with the unique non-spurious solution $A^{(\mathrm{EEP})}=6$ and $B^{(\mathrm{EEP})}=4$.

\section{Six-dimensional case}

The secular equation at $N=6$,

$\operatorname{det}\left[\begin{array}{cccccc}5-E & c & 0 & 0 & 0 & 0 \\ -c & 3-E & b & 0 & 0 & 0 \\ 0 & -b & 1-E & a & 0 & 0 \\ 0 & 0 & -a & -1-E & b & 0 \\ 0 & 0 & 0 & -b & -3-E & c \\ 0 & 0 & 0 & 0 & -c & -5-E\end{array}\right]=0$

in its polynomial form for $s=E^{2}$,

$s^{3}+\left(2 b^{2}-35+2 c^{2}+a^{2}\right) s^{2}$

$+\left(b^{4}+2 c^{2} a^{2}-44 b^{2}+28 c^{2}-34 a^{2}+c^{4}+259+2 b^{2} c^{2}\right) s$

$+a^{2} c^{4}-10 b^{2} c^{2}+30 c^{2} a^{2}+225 a^{2}-30 c^{2}-c^{4}-25 b^{4}$

$-225-150 b^{2}=0$

is still solvable in closed form. In the EEP extreme the full solution of the triplet of eqs. (9) ceases to be easy but it still remains feasible, giving

$A^{(\mathrm{EEP})}=9, \quad B^{(\mathrm{EEP})}=8, \quad C^{(\mathrm{EEP})}=5, \quad N=6$.

\section{Seven-dimensional case}

By the same Gröbner-basis method as above we derive the result

$A^{(\mathrm{EEP})}=12, \quad B^{(\mathrm{EEP})}=10, \quad C^{(\mathrm{EEP})}=6, \quad N=7$.

It is again unique because one of the two roots $C_{ \pm}=27 \pm 9 \sqrt{21}$ of the "first alternative" Gröbnerian "effective" equation $C^{2}-54 C=972$ and both the roots $-354 \pm 60 \sqrt{34}$ of the "second alternative" equation $C^{2}+708 C+2916=0$ are negative, while the only remaining positive root $C_{+}=68.24318125$ gives the negative $B=28-3 C$.

\section{Extrapolation formulae of paper [19]}

By construction, quasi-Hermiticity domain $\mathcal{D}$ must lie inside a simplex $S$,

$A+2(B+C+\ldots+Z) \equiv g_{J}^{2}+2 \sum_{k=1}^{J-1} g_{k}^{2} \leq \frac{4 K^{3}-K}{3}$,

$N=2 K=$ even

and

$A+B+C+D+\ldots+Z \leq \frac{2 M^{3}+3 M^{2}+M}{3}$,

$N=2 M+1=$ odd .
This provides important information about the shape of the domain $\mathcal{D}$, obtained fairly easily by the extrapolation technique (cf. [19]).

In the light of the simplicity of all our previous EEP formulae the extrapolation trick can be applied to them as well. At the even $N=2 K$ such an approach leads to the extrapolation conjecture

$$
\begin{aligned}
& A^{(\mathrm{EEP})}=K^{2}, \quad B^{(\mathrm{EEP})}=K^{2}-1^{2}, \\
& C^{(\mathrm{EEP})}=K^{2}-2^{2}, \quad D^{(\mathrm{EEP})}=K^{2}-3^{2}, \quad \ldots
\end{aligned}
$$

the validity of which we tested up to $K=6$. In parallel one arrives at the following odd-dimensional formula

$A^{(\mathrm{EEP})}=M(M+1)$,

$B^{(\mathrm{EEP})}=M(M+1)-1 \cdot 2=M(M+1)-2$,

$C^{(\mathrm{EEP})}=M(M+1)-2 \cdot 3$,

$D^{(\mathrm{EEP})}=M(M+1)-3 \cdot 4, \ldots$

when $N=2 M+1$.

\section{Verifications: Eight-dimensional case}

Even dimensions $N=2 K$ are "anomalous" in having the coupling $a$ in the matrix

$H^{(2 K)}=\left[\begin{array}{cccc|cccc}2 K-1 & z & 0 & \cdots & & & & \\ -z & \ddots & \ddots & \ddots & \vdots & & & \\ 0 & \ddots & 3 & b & 0 & \cdots & & \\ \vdots & \ddots & -b & 1 & a & 0 & \cdots & \\ \hline & \cdots & 0 & -a & -1 & b & 0 & \cdots \\ & & \cdots & 0 & -b & -3 & \ddots & \\ & & & \vdots & \ddots & \ddots & \ddots & z \\ & & & & \cdots & 0 & -z & 1-2 K\end{array}\right]$

just once. This is reflected by the specific, "anisotropic" form of the simplex (15).

Once we had revealed the general $N$-dependence of the similar formulae, it was necessary to test these conjectures. The eight by eight model with $K=4$ played a key role in it since the complexity of its non-numerical description is already quite perceivable. The situation is still not bad when the circumscribed simplex with the definition

$$
A+2 B+2 C+2 D=84
$$

is sought. The subsequent EEP construction is much more difficult. It is based on simultaneous solution of the quadratic, cubic and quartic polynomial equations $P_{2}(A, B, C, D)=0$, $P_{1}(A, B, C, D)=0$, and $P_{0}(A, B, C, D)=0$ containing 13,19 and 20 individual terms, respectively. Just a marginal simplification exists, e.g., in the $\mathrm{P}_{2}$ - case reducible to the 9-term equation

$$
\begin{aligned}
1974+ & (B+C+D)^{2}+2 A D+2 B D+2 A C \\
= & 83 A+142 B+70 C-50 D
\end{aligned}
$$

Still, a decisive formal merit of our model is reflected by the survival of the simplicity of the final formula

$A^{(\mathrm{EEP})}=16, B^{(\mathrm{EEP})}=15, C^{(\mathrm{EEP})}=12, D^{(\mathrm{EEP})}=7, N=8$. 
One can only appreciate the latter observation when one sees the final Gröbner-basis element which defines $D^{(\text {EEP })}=7$ as a root of the following seventeenth-degree polynomial

$314432 D^{17}-5932158016 D^{16}+4574211144896 D^{15}+3133529909492864 D^{14}+917318495163561932 D^{13}$

$+167556261648918275684 D^{12}+14670346929744822064505 D^{11}+720991093724510065469933 D^{10}$

$+62429137451114251409236415 D^{9}+676326278232758784369966787 D^{8}+40525434802944282153115803370 D^{7}$

$+2361976444746440513605248930610 D^{6}-145759836636885012145070948315366 D^{5}$

$+8129925258122948689157916436170874 D^{4}-68875673245487669398850290405642067 D^{3}$

$+235326754101824439936800228806905073 D^{2}-453762279414621179815552897029039797 D$

$+153712881941946532798614648361265167=0$

In a test of the uniqueness of solution (19) one finds out that it possesses seven real and positive roots $D$. Out of these, the following three of them are negative and, hence, manifestly spurious,-203.9747095, -156.6667001, -55.49992441 . The proof of the spuriosity for the remaining four roots $0.4192854385,5.354156128,1354.675195$ and, however straightforward, becomes unpleasant and clumsy. For example, the values of $A$ are given by the rule $\alpha \times a=$ (a polynomial in $D$ of 16th degree) where the number of digits in the auxiliary integer constant $\alpha$ exceeds one hundred.

\section{Odd dimensions and the test at $N=9$}

It remains for us to discuss the models with odd dimensions $N=2 M+1$,

$H^{(2 M+1)}=\left[\begin{array}{ccc|c|ccc}2 M & z & 0 & 0 & 0 & 0 & 0 \\ -z & \ddots & \ddots & 0 & 0 & 0 & 0 \\ 0 & \ddots & 2 & a & 0 & 0 & 0 \\ \hline 0 & 0 & -a & 0 & a & 0 & 0 \\ \hline 0 & 0 & 0 & -a & -2 & \ddots & 0 \\ 0 & 0 & 0 & 0 & \ddots & \ddots & z \\ 0 & 0 & 0 & 0 & 0 & -z & -2 M\end{array}\right]$

and to test and verify the validity of the extrapolated formulae at $N \geq 9$.

At $M=4$ we were still able to evaluate the explicit form of the secular equation,

$$
\begin{aligned}
& 14745600-7372800 A+\ldots \\
& +(-2 C+220-2 B-2 A-2 D) s^{4}-s^{5}=0
\end{aligned}
$$

and to re-derive the expected $M=4$ EEP values by its direct solution,

$A^{(\mathrm{EEP})}=20, \quad B^{(\mathrm{EEP})}=18, \quad C^{(\mathrm{EEP})}=14$, $D^{(\mathrm{EEP})}=8, \quad N=9$.

At a few higher $M>4$ we just re-confirmed the validity of the extrapolated formulae (18) by their insertion in secular equations.

\section{Acknowledgment}

This work has been supported by the MŠMT Doppler Institute project Nr. LC06002, by the Institutional Research Plan AV0Z10480505 and by GAČR grant Nr. 202/07/1307.

\section{References}

[1] Styer, D. F. et al.: Nine Formulations of Quantum Mechanics, Amer. J. Phys. Vol. 70 (2002), p. 288.

[2] Scholtz, F. G., Geyer, H. B., Hahne, F. J. W.: Quasi-Hermitian Operators in Quantum Mechanics and the variational Principle, Ann. Phys. (NY), Vol. 213 (1992) p. 74-101.

[3] cf. http://www.mth.kcl.ac.uk/ streater/lostcauses.html\#XIII

[4] Bender, C. M., Boettcher, S.: Real Spectra in non-Hermitian Hamiltonians Having $\mathcal{P}$ T-Symmetry, Phys. Rev. Lett. Vol. 80 (1998), p. 5243-5246.

[5] Bender, C. M., Turbiner, A.: Analytic Continuation of Eigenvalue Problems, Phys. Lett. A Vol. 173 (1993), p. 442; A. Turbiner, private communication (April/May, 2000).

[6] Buslaev, V., Grecchi, V.: Equivalence of Unstable Anharmonic Oscillators and Double Wells, J. Phys. A: Math. Gen. Vol. 26 (1993), p. 5541-5549; V. Grecchi, private communication (February, 2000).

[7] Znojil, M.: Should PT-Symmetric Quantum Mechanics be Interpreted as Nonlinear?, J. Nonlin. Math. Phys. Vol. 9, suppl. 2 (2002), p. 122-133 (quant-ph/0103054).

[8] Znojil, M.: Conservation of Pseudo-Norm in PT-Symmetric Quantum Mechanics, Rendiconti del Circ. Mat. di Palermo, Ser. II, Suppl. 72 (2004), p. 211-218, (math-ph/0104012).

[9] Bagchi, B., Quesne, C., Znojil, M.: Generalized Continuity Equation and Modified Normalization in $\mathcal{P}$-Symmetric Quantum Mechanics, Mod. Phys. Lett. A, Vol. 16 (2001), p. 2047-2057.

[10] Mostafazadeh, A.: Pseudo-Hermiticity Versus PT Symmetry: The Necessary Condition for the Reality of the Spectrum of a non-Hermitian Hamiltonian, J. Math. Phys. Vol. 43 (2002), p. 205-214.

[11] Mostafazadeh, A.: Pseudo-Hermiticity Versus PT-Symmetry II. A complete Characterization of non-Hermitian Hamiltonians with a Real Spectrum, J. Math. Phys. Vol. 43 (2002), p. 2814-2816;

[12] Mostafazadeh, A.: Pseudo-Hermiticity Versus PT-Symmetry III: Equivalence of pseudo-Hermiticity and the Presence of Antilinear Symmetries, J. Math. Phys. Vol. 43 (2002), p. 3944-3951;

[13] Langer, H., Tretter, Ch.: A Krein Space Approach to PT-Symmetry, Czech.J. Phys. Vol. 54 (2004), p. 1113-1120. 
[14] Bender, C. M., Brody, D. C., Jones, H. F.: Complex Extension of Quantum Mechanics, Phys. Rev. Lett. Vol. 89 (2002), 0270401; Erratum-ibid. 92 (2004), 119902.

[15] Znojil, M., Geyer, H. B.: Construction of a Unique Metric in quasi-Hermitian Quantum Mechanics: Nonexistence of the Charge Operator in a $2 \times 2$ Matrix Model, Phys. Lett. B, Vol. 640 (2006) 52-56; Erratum-ibid, Phys. Lett. B 649 (2007) - see [16] below.

[16] In ref. [15] we accepted a specific overall scale $Z=1$ in $\Theta$. In the resulting one-parametric subfamily $\Theta$ of the factorization postulate $\Theta=\mathcal{C P}$ of ref. [14] led to the charge factor $\mathrm{C}$, which was not involutive. In order to avoid possible misunderstandings we would like to emphasize here that neither the involutivity $\mathcal{C}^{2}=I$ nor the preference of $Z=1$ are based on deeper physical grounds. In this sense, the statements formulated in the last four lines of paragraph 3.2 of ref. [15] (plus their several citations throughout the text) should be interpreted with due care. It is obvious that, whenever necessary, one can always return to the full, two-parametric family of $\Theta$ and achieve the involutivity of charge $\mathcal{C}$ via an elementary Hamiltonian-dependent adaptation of the scale $Z=Z(H)$.

[17] Znojil, M.: A Return of Observability near Exceptional Points in a Schematic PT-Symmetric Model, Phys. Lett. B, Vol. 647 (2007), p. 225-230.
[18] Znojil, M.: Determination of the Domain of the Admissible Matrix Elements in the Four-Dimensional PT-Symmetric Anharmonic Model, Phys. Lett. A, Vol. 367 (2007), p. 300-306.

[19] Znojil, M.: Maximal Couplings in PT-Symmetric Chain-Models with the Real Spectrum of Energies, J. Phys. A: Math. Theor. Vol. 40 (2007), p. 4863-4875, (math-ph/0703070v1)

[20] Znojil, M.: Conditional Observability, Phys. Lett. B, Vol. 650 (2007), p. 440-446 (arXiv:0704.3812v1 [math-ph]).

[21] Bender, C. M.: Making Sense of non-Hermitian Hamiltonians, Rep. Prog. Phys., submitted (hep-th/0703096 and preprint LA-UR-07-1254).

[22] Kato, T.: Perturbation Theory for Linear Operators Berlin: Springer, 1966, p. 64.

Miloslav Znojil, DrSc.
phone: +420266 173286
e-mail: znojil@ujf.cas.cz

Academy of Science of the Czech Republic

Nuclear Physics Institute

25068 Řež, Czech Republic 